Revue internationale P.M.E.

Économie et gestion de la petite et moyenne entreprise

\title{
Difficultés de gestion et besoins de formation des dirigeants des TPE en démarrage
}

\section{Alain Capiez}

Volume 3, numéro 3-4, 1990

URI : https://id.erudit.org/iderudit/1007984ar

DOI : https://doi.org/10.7202/1007984ar

Aller au sommaire du numéro

Éditeur(s)

Presses de l’Université du Québec

ISSN

0776-5436 (imprimé)

1918-9699 (numérique)

Découvrir la revue

Citer cet article

Capiez, A. (1990). Difficultés de gestion et besoins de formation des dirigeants des TPE en démarrage. Revue internationale P.M.E., 3(3-4), 327-344.

https://doi.org/10.7202/1007984ar
Résumé de l'article

Le succès d'une Très Petite Entreprise (TPE) en démarrage repose essentiellement sur son dirigeant et sur sa capacité à concevoir une stratégie adaptée à son marché. La formation du dirigeant joue ainsi un rôle primordial. C'est pourquoi nous avons réalisé une enquête sur 29 TPE en démarrage, afin de mettre en évidence les difficultés de gestion que leurs dirigeants avaient rencontrées et les besoins de formation qu'ils pouvaient ressentir. Il apparaît que, dans l'ensemble, les besoins de formation suivent les problèmes rencontrés, mais avec un certain décalage correspondant au temps nécessaire à la prise de conscience par le dirigeant du besoin d'une formation approfondie, afin de gérer efficacement une organisation dont la complexité s'intensifie avec le temps.
Tous droits réservés @ Presses de l’Université du Québec, 1990
Ce document est protégé par la loi sur le droit d'auteur. L'utilisation des services d’Érudit (y compris la reproduction) est assujettie à sa politique d'utilisation que vous pouvez consulter en ligne.

https://apropos.erudit.org/fr/usagers/politique-dutilisation/ 


\title{
Difficultés de gestion et besoins de formation des dirigeants des TPE en démarrage
}

\author{
Alain CAPIEZ* \\ Université d'Angers
}

\begin{abstract}
RÉSUMÉ
Le succès d'une Très Petite Entreprise (TPE) en démarrage repose essentiellement sur son dirigeant et sur sa capacité à concevoir une stratégie adaptée à son marché. La formation du dirigeant joue ainsi un rôle primordial. C'est pourquoi nous avons réalisé une enquête sur 29 TPE en démarrage, afin de mettre en évidence les difficultés de gestion que leurs dirigeants avaient rencontrées et les besoins de formation qu'ils pouvaient ressentir. II apparaît que, dans l'ensemble, les besoins de formation suivent les problèmes rencontrés, mais avec un certain décalage correspondant au temps nécessaire à la prise de conscience par le dirigeant du besoin d'une formation approfondie, afin de gérer efficacement une organisation dont la complexité s'intensifie avec le temps.
\end{abstract}

\begin{abstract}
The success of a Smaller Business Firm (SBF) rests essentially with its manager; for a manager, his ability to fit his strategy with the market's characteristics is fundamental. So the training of the manager is of prime importance. This article presents the results of an inquiry about 29SBF in early stage of development and points out the main management problems and the most important training needs their managers experienced. On the whole, it appears that the training needs are following the management problems, yet there is some lag. This lag is the time the manager needs to realize that a complete training is the only way to successfully manage an organisation getting more and more intricate as time is going on.
\end{abstract}

* Adresse : Académie de Nantes, Université d'Angers, Faculté de Droit, d'Économie et des Sciences sociales, 11, boul. Lavoisier, 49045 Angers, Cedex, France. Premier manuscrit reçu, février 1990. 


\section{RESUMEN}

El exito de una Empresa Muy Pequena (EMP) en la etapa de puesta en marcha, se fundamenta en su dirigente y en su capacidad para elaborar una estrategia que se acomoda con su mercado. Por eso, la formacion de su dirigente desempena un gran papel. Este articulo presenta los resultados de una encuesta sobre 29 EMP en la etapa de puesta en marcha. Gracia a su analisis, hemos podido determinar los principales problemas de gestion y los esenciales necessidades de formacion que sus dirigentes encontraron. En general, los necessidades de formacion sigen los problemas de gestion, pero hay una diferencia. Esta diferencia permite que el dirigente se da cuenta de la necessidad de una formacion detenida, para que lleva bien una organizacion que se complica con il tiempo. 


\section{Introduction}

De nombreuses études ont mis en évidence l'importance du rôle du dirigeant dans l'émergence d'une toute petite entreprise (TPE); la formation que ce dirigeant a reçue et qu'il recevra est donc d'une importance primordiale.

Plus particulièrement, pour les TPE récemment créées, en phase de démarrage, il est important d'identifier les liens entre les problèmes de gestion rencontrés et les besoins de formation complémentaire qu'ils entraînent chez leurs dirigeants, afin qu'ils puissent mener à bien leur entreprise en devenant des managers de plus en plus efficaces. C'est pourquoi nous avons réalisé, d'octobre 1988 à avril 1989, une enquête sur une trentaine d'entreprises créées à la suite de stages créateurs organisés de 1985 à 1988, et portant sur les difficultés de gestion et les besoins de formation de leurs dirigeants.

Après avoir présenté la méthodologie de l'enquête et les caractères de l'échantillon, nous étudierons les problèmes de gestion des TPE en démarrage et les besoins de formation complémentaires exprimés par leurs dirigeants, puis nous rechercherons les liens entre les difficultés de gestion et les besoins de formation.

\section{Méthodologie et échantillon}

Le démarrage est une phase essentielle du cycle de vie de l'entreprise. A la suite des travaux de A. Marshall à la fin du siècle dernier, N.C. Churchill et V.L. Lewis (1983) ont distingué cinq stades d'évolution d'une PME : l'existence, le maintien, la réussite, l'essor et l'exploitation maximale des ressources; M. Marchesnay (1986), plus proche du modèle d'A. Marshall, propose la création, le démarrage et la montée en compétitivité, le régime de croisième et développement, le déclin et le retrait. La phase de démarrage regroupe les stades successifs d'existence et de maintien et peut durer de deux à cinq ans. Les principaux problèmes de gestion qui se posent pendant le démarrage ont été étudiés par H. Mahe (1984), hiérarchisés par C. Fourcade (1988) et synthétisés par G. D'Amboise (G. D'Amboise et A. Parent, 1988). Les besoins de formation complémentaire qui en résultent ont été étudiés d'abord de façon empirique et simple (J. Thomas et $\mathrm{P}$. Sireno, 1981), puis de manière plus analytique (B. Garnier, Y. Gasse et P. Cossette, 1984, H. Mahe, 1985).

Dans le prolongement de ces études, nous avons cherché à mettre en évidence les liens entre difficultés de gestion et besoins de formation des TPE en démarrage, à l'aide d'une enquête par questionnaire, pour laquelle nous présenterons la méthodologie utilisée et les caractéristiques de l'échantillon.

\subsection{Méthodologie de l'enquéte}

Nous sommes partis d'une population de 104 personnes ayant suivi un stage de création d'entreprise organisé par le Centre Permanent de Formation à la Création 
d'Entreprise (C.P.F.C.E.) de la Chambre des Métiers de Maine-et-Loire, entre mai 1985 et juin 1988. Sur ces 104 personnes, 39 stagiaires avaient effectivement créé une entreprise, encore en activité en février 1989. Nous leur avons envoyé par la poste un questionnaire détaillé (8 pages) permettant de préciser les difficultés rencontrées au cours de la période de démarrage et les besoins en formation qui peuvent en résulter. Les réponses obtenues ont été complétées par des entretiens téléphoniques. Au total, 29 questionnaires complets et exploitables ont été retenus; ils constituent notre échantillon. Le questionnaire était divisé en cinq parties :

1- Caractéristiques de l'entreprise

2- Difficultés de gestion

3- Relations avec les partenaires

4- Gestion du temps du dirigeant

5- Besoins de formation

Les parties les plus importantes étaient les parties 2 et 5 ; elles seules constituent l'objet de cette étude et seront analysées ici. Dans la partie 2, il était demandé au dirigeant d'indiquer son degré d'accord avec 21 propositions relatives aux difficultés de gestion rencontrées par les dirigeants; le dirigeant devait répondre sur une échelle de Likert allant de 1 (pas du tout) à 5 (totalement); toutes ces propositions étaient rattachées à une des grandes fonctions suivantes : Marketing (1 à 7), Production (8 à 11), Finances (12 à 17), Personnel (19 à 21), mais le regroupement n'était pas indiqué. Dans la partie 5, il était demandé au dirigeant d'indiquer pour 43 domaines précis de gestion, l'importance de l'aide qu'une formation spécifique lui apporterait pour la gestion de son entreprise; il devait répondre sur une échelle de Likert allant de 1 (nulle) à 5 (fondamentale); tous ces domaines étaient rattachés à une des grandes fonctions suivantes : Administration générale (1 à 13 et 40), Comptabilité (14,15, 17), Finances (18 à 25 et 16), Marketing (26 à 33), Production (34 à 38), Personnel $(39,41,42,43)$, mais le regroupement n'était pas indiqué, là non plus.

Une analyse uni-dimensionnelle portant sur les scores moyens obtenus pour chaque proposition et pour chaque fonction de l'entreprise concernée permettra, dans un premier temps, d'évaluer les principaux problèmes de gestion posés au créateur dans la phase de démarrage de son affaire, ainsi que les besoins de formation qui lui apparaissent les plus pressants.

Dans un deuxième temps, une analyse multi-dimensionnelle sera effectuée afin de mettre en évidence les relations entre problèmes de gestion et besoins de formation. Pour cela, nous reprendrons les quatre variables de problèmes définis précédemment pour chaque fonction : problèmes de marketing (pmk), problèmes de finances (pfi), problèmes de production (ppr) et problèmes de personnel (ppl), et les six variables de besoins définies pour chaque fonction : besoins en administration générale (bag), besoins en marketing (bmk), besoins en finances (bfi), besoins en comptabilité (bcp), besoins en production (bpr) et besoins en personnel (bpl). Nous chiffrerons de manière ordinale l'intensité de ces problèmes et de ces besoins à partir 
des scores obtenus, selon cinq modalités : 1 = problèmes ou besoins inexistants, 2 = problèmes ou besoins faiblement ressentis, 3 = problèmes ou besoins moyennement ressentis, 4 = problèmes ou besoins fortement ressentis, $5=$ problèmes ou besoins fondamentaux, ceci pour chaque entreprise de notre échantillon.

\subsection{Caractères généraux de l'échantillon}

Notre échantillon est composé de 29 TPE en démarrage, réparties en quatre groupes d'âge, en fonction de leur durée d'existence à la fin de février 1989, et notés a1 = âge 1 (moins de 6 mois) ( 4 entreprises), a2 = âge 2 (6 mois à 1 an) (14 entreprises), a3 = âge 3 ( 1 à 2 ans) ( 7 entreprises), a4 = âge 4 ( 2 à 3 ans) (4 entreprises), et en deux secteurs notés : sct1 = secteur 1 (entreprises du bâtiment, de services et du commerce), sct 2 = secteur 2 (entreprises de production transformatrices).

Les formes juridiques adoptées sont en majorité l'entreprise individuelle (13) et la S.A.R.L. (11), 5 entreprises ayant toutefois choisi la forme récente d'E.U.R.L. ${ }^{1}$. Les dirigeants oeuvrent dans 11 secteurs différents, 8 entreprises appartenant à l'industrie au sens strict, 6 à l'industrie du bâtiment, 5 au commerce et 10 aux services. La taille de ces entreprises est très petite, 17 n'ayant aucun salarié, 8 employant de 2 à 5 salariés et 3 employant 6, 7-1/2 et 8 salariés. En ce qui concerne le niveau d'études de leurs dirigeants-propriétaires, c'est le secondaire qui prédomine (76\%), et en particulier le secondaire technique (59\%), et pour le reste, le primaire (10\%) et le supérieur (14\%). En outre, la quasi-totalité des créateurs de notre échantillon (27) possède une expérience professionnelle préalable à la création, de durée moyenne de 12 ans, avec une qualification, essentiellement de niveau ouvrier (10) et employé (8), mais aussi de technicien et agent de maîtrise (6) et même de cadre (3). Tous ont suivi un stage de 3 mois de formation intensive à la gestion avant de créer leur entreprise. Il est évident que les candidats à la création d'entreprise ont été sélectionnés par le centre de formation, en fonction de la qualité et de l'avancement de leurs projets; aussi un taux de création d'entreprise, calculé sur cet échantillon, serait-il fortement biaisé. Mais nous nous intéressons uniquement ici aux problèmes posés dans la phase de démarrage de la TPE; notre échantillon est alors tout à fait représentatif de TPE récemment créées, avec un projet solide et opérationnel lors de la création, et un créateur possédant, au minimum, une formation de base en économie et en gestion. Il s'agit véritablement d'entreprises de taille toute petite (moins de 10 salariés), inférieure à celle de la plupart des échantillons habituellement étudiés. La limite à une généralisation des résultats de cette enquête provient de la taille limitée de l'échantillon; aussi comparerons-nous nos résultats avec ceux d'autres études voisines, chaque fois que cela sera possible.

1 La forme juridique de «société» permet de limiter la responsabilité des associés à leurs seuls apports. La Société à Responsabilité Limitée (S.A.R.L.) ne demande que 2 associés au minimum contre 7 pour la Société Anonyme (S.A.). L'Entreprise Unipersonnelle à Responsabilité Limitée (E.U.R.L.), créée en France par la loi du 11-07-85, est une variante de la S.A.R.L. avec un «associé unique». 
L'analyse uni-dimensionnelle des résultats fait apparaître les principaux problèmes de gestion posés ainsi que les demandes de formation complémentaires.

\section{Problèmes de gestion et besoins de formation complémentaires}

L'examen des scores moyens obtenus pour chaque proposition puis pour chaque fonction permet de hiérarchiser les problèmes de gestion des TPE en démarrage ainsi que les besoins de formation complémentaires ressentis par leurs dirigeants.

\subsection{Problèmes de gestion des micro-firmes en démarrage}

Les principaux problèmes de gestion rencontrés par les dirigeants des TPE en démarrage sont récapitulés dans le tableau 1, p. 334.

Le problème fondamental qui conditionne l'existence même de l'entreprise est la recherche de nouveaux clients : recherche d'une clientèle suffisante pour absorber les premières productions dans l'entreprise de moins de six mois (groupe a1), recherche de clients supplémentaires pour obtenir une base de vente suffisante, afin de garantir l'avenir de l'affaire, sa pérennité, dans les autres groupes. Cette recherche s'accompagne de l'analyse de la clientèle, laquelle n'a pas toujours été sélectionnée soigneusement lors de la première année d'activité où la recherche l'emporte sur le choix; plusieurs entreprises signalent d'ailleurs des relations difficiles avec un ou plusieurs gros clients (avec un score de 1.79). Tout cela ne va pas sans difficultés dans un univers fortement concurrentiel.

C'est la menace omniprésente d'une concurrence active qui constitue le principal risque de marché pour les dirigeants de notre échantillon, et ce, d'autant plus que l'entreprise se développe. Par contre, il semble que les dirigeants aient plus de mal à apprécier l'étendue de leur marché qui, large, demande une expansion rapide pour acquérir un pouvoir de marché, et étroit, rend l'entreprise dépendante de quelques gros clients. C'est cependant des risques de l'étroitesse du marché que les dirigeants ont le plus conscience (score de 1,52 contre 1,38 pour le marché trop large), l'un d'eux notant: " comment envisager l'avenir avec $80 \%$ en sous-traitance pour un gros client?», et d'autres, indiquant des difficultés avec de gros clients, comme nous l'avons déjà remarqué.

Lorsque l'entreprise a obtenu une clientèle suffisante pour assurer sa viabilité, il lui faut la satisfaire avec des biens ou des services, adaptés aux besoins de ces clients et produits dans de bonnes conditions. La recherche de nouveaux fournisseurs est un moyen d'atteindre cet objectif, plus particulièrement dans les entreprises de plus d'un an d'existence; un dirigeant précise : «il faut trouver des pièces de rechange au bon endroit et au moment voulu». L'autre moyen est de 
résoudre les problèmes de choix technologiques: fabriquer les produits à temps et en quantité voulue (8e difficulté) et choisir les procédés de fabrication appropriés (10 difficulté). Mais les investissements nécessaires à l'adaptation de la capacité de production sont souvent trop lourds pour le budget des petites entreprises en démarrage ( $5^{\mathrm{e}}$ difficulté) qui souffrent d'une insuffisance de fonds propres et de possibilités d'emprunts limitées. Si cette dernière difficulté obtient un score assez faible, c'est que l'horizon de prévision du dirigeant est limité à un ou deux ans maximum, en raison de la multitude des problèmes à résoudre pendant cette phase; si le dirigeant a conscience des besoins futurs d'investissement, il prévoit assez mal le montant des ressources financières. Par contre, il est préoccupé par la faiblesse de la rentabilité.

- L'insuffisance de la rentabilité vient en quatrième position. Pendant les six premiers mois, il faut couvrir les frais considérables entraînés par le lancement de l'activité; à la fin du premier exercice, les comptes sont établis les comptes et le premier résultat se révèle souvent négatif. Au cours du deuxième exercice se pose la question de savoir si le cash flow est suffisant pour reconstituer l'actif immobilisé, afin de rester en activité, et, au-delà, de financer une croissance suffisante. Le dirigeant prend conscience du coût des emprunts et du poids des charges financières sur son résultat d'exploitation. Et pendant tout ce temps, il faut faire face à ses échéances chaque jour.

- La difficulté à assurer ses échéances est classée en sixième position. Dès le départ, le dirigeant va rechercher les aides à la création et divers prêts; les aides à la création, souvent accordées selon des critères très pointilleux et liquidés tardivement, sont fréquemment qualifiés de «miroir aux alouettes» par les dirigeants désillusionnés. Quant aux prêts, ils peuvent être subordonnés à des garanties personnelles et/ou à un montage de dossier que ne peut pas toujours réaliser correctement le dirigeant. Retards et non-versements ne facilitent pas les règlements. Mais les difficultés de trésorerie viennent essentiellement d'un besoin structurel en fonds de roulement important, difficulté classée neuvième dans notre échantillon; en effet, les fournisseurs exigent souvent un paiement rapide de la jeune entreprise qui leur semble peu fiable, alors que les clients ont tendance à abuser des délais de paiement, et ce, d'autant plus qu'ils constituent une partie importante du chiffre d'affaires. Les crédits bancaires à court terme sont alors indispensables, mais leurs coût pèse lourdement sur la rentabilité de l'entreprise.

- Remarquons que les relations avec l'Administration et les organismes consulaires entraînent un certain agacement (score de 1,93), surtout pendant les six premiers mois où certaines formalités de la période de création peuvent encore se poursuivre; autrement, c'est un certain irréalisme qui est reproché à ses organisations.

- Enfin, les problèmes de personneln'apparaissent pas fondamentaux pour les entreprises de notre échantillon dont $59 \%$ n'ont aucun salarié; certaines fonctionnent avec deux ou trois associés qui n'ont eu aucune difficulté à définir leur rôle et à se 
Tab. 1 Les dix problèmes de gestion les plus importants selon les dirigeants

\begin{tabular}{|c|c|c|c|c|c|c|}
\hline & \multirow[b]{2}{*}{ Problèmes de gestion } & \multicolumn{5}{|c|}{ Score moyen (max. : 5) } \\
\hline & & groupe & groupe & groupe & groupe & Ensem- \\
\hline 1 & Vous recherchez de nouveux clients & 4,25 & 4,43 & 4,29 & 4,50 & 4,38 \\
\hline 2 & La concurrence est très active sur votre marché & 2,50 & 3,14 & 4,14 & 4,25 & 3,44 \\
\hline 3 & Vous recherchez de nouveaux foumisseurs & 1,25 & 2,64 & 4,00 & 3,00 & 2,83 \\
\hline 4 & Votre rentbilité est insuffisante & 2,00 & 2,71 & 2,43 & 3,00 & 2,59 \\
\hline 5 & $\begin{array}{l}\text { Les investissements nécessaires sont trop lourds pour votre } \\
\text { budget }\end{array}$ & 2,00 & 1,86 & 2,86 & 3,25 & 2,34 \\
\hline 6 & Les emprunts vous coatent trop cher & 1,75 & 1,71 & 3,00 & 3,75 & 2,31 \\
\hline 7 & Vous avez des diifficultés à assurer vos échéances & 3,25 & 2,07 & 2,00 & 2,25 & 2,24 \\
\hline 8 & $\begin{array}{l}\text { Vous avez des difficultés à assurer la livraison de vos } \\
\text { produits à temps et en quantité voulue (entreprises de } \\
\text { production) }\end{array}$ & & 1,67 & 3,00 & & 2,14 \\
\hline $\mathbf{9}$ & $\begin{array}{l}\text { Vous n'obtenez pas de vos fournisseurs des délais } \\
\text { compatibles avec les délais de crédit accordés à vos clients }\end{array}$ & 1,50 & 1,93 & 2,29 & 2,67 & 2,06 \\
\hline $\begin{array}{l}1 \\
0\end{array}$ & $\begin{array}{l}\text { Le choix des procédés de fabrication vous pose des } \\
\text { difficultés (entreprises de production) }\end{array}$ & & 1,67 & 2,67 & & 2,05 \\
\hline
\end{tabular}

Tab. 2 Les problèmes de gestion regroupés par fonction

\begin{tabular}{|c|c|c|c|c|c|}
\hline \multirow{2}{*}{ Fonction } & \multicolumn{5}{|c|}{ Score moyen (max. :5) } \\
\hline & $\begin{array}{c}\text { groupe } \\
1\end{array}$ & \begin{tabular}{|c|} 
groupe \\
2
\end{tabular} & $\begin{array}{c}\text { groupe } \\
3\end{array}$ & $\begin{array}{c}\text { groupe } \\
4\end{array}$ & $\begin{array}{c}\text { Ensem- } \\
\text { ble }\end{array}$ \\
\hline $\begin{array}{l}\text { Vente et marketing } \\
\text { (marché, clients, fournisseurs) }\end{array}$ & 1,93 & 2,27 & 2,65 & 2,64 & 2,37 \\
\hline Finances & 1,89 & 2,17 & 2,65 & 2,94 & 2,35 \\
\hline Production et technique & 1,00 & $\begin{array}{r}1,47 \\
1,63^{*}\end{array}$ & $\begin{array}{r}1,71 \\
2,49^{*}\end{array}$ & 1,18 & $\begin{array}{r}1,42 \\
2,12^{*}\end{array}$ \\
\hline Personnel & 1,00 & 1,51 & 1,53 & 1,16 & 1,40 \\
\hline Score global & 1,46 & 1,86 & 2,14 & 1,98 & 1,89 \\
\hline
\end{tabular}

- Entreprises de production transformatices 
répartir les tâches. La préoccupation la plus importante en ce domaine reste la recherche de personnel qualifié pour l'avenir immédiat et plus lointain.

En regroupant ces problèmes par fonction (voir tableau 2), c'est la «Vente et Marketing» qui vient en tête (score $=2,37$ ) puis les «Finances» $(=1,40)$. Le tableau 2 fait apparaître l'évolution de l'intensité des problèmes selon les groupes d'entreprises. Les problèmes financiers et les problèmes de marketing en particulier semblent évoluer parallèlement et croître avec l'âge; cette évolution sera étudiée de manière plus approfondie par l'analyse multi-dimensionnelle des données.

Tous ces problèmes de gestion, auxquels le dirigeant se trouve confronté, sont à l'origine de besoins de formation venant compléter la formation reçue préalablement à la création.

\subsection{Besoins de formation complémentaire des dirigeants de micro-firmes en démarrage}

Le tableau 3, p. 236 indique les dix (douze avec les ex aequo) besoins de formation les plus importants selon les dirigeants. On remarque que six de ces besoins sont des besoins financiers $(2,5 \mathrm{~b}, 8,9,10 \mathrm{a}, 10 \mathrm{~b})$; on peut penser que les problèmes financiers font appel à des techniques complexes encore insuffisamment maîtrisées par les dirigeants d'entreprise en démarrage, de sorte que si les problèmes financiers arrivent en deuxième position, les besoins de formation financière, eux, sont en première position. En dehors de l'attendu "comment payer moins d'impôts?», on trouve la négociation avec le banquier, la gestion de trésorerie et la prévision des besoins en fonds de roulement, les prévisions de rentabilité et la détermination et le contrôle des objectifs financiers. Trois besoins ( $3 \mathrm{a}, 3 \mathrm{~b}, 10 \mathrm{c}$ ) appartiennent ensuite à la fonction «Marketing» où se posent les problèmes les plus intensément ressentis : il s'agit en toute logique de la recherche des besoins des clients, de la détermination d'un plan d'action commerciale et de moyens publicitaires efficaces. Trois besoins relèvent de la fonction «Administration Générale» $(1,5 \mathrm{a}, 7)$; deux d'entre eux sont directement liés aux relations avec les partenaires commerciaux; ils peuvent se résumer en trois termes qui caractérisent le bon dirigeant : efficacité, négociation, communication.

Sil'on recherche l'intensité des besoins de formation par fonction (voir tableau 4, p. 236), ce sont les fonctions «Finances» et «Marketing» qui apparaissent en tête; la fonction «Comptabilité» figure en troisième position avec un score nettement inférieur à celui de la finance, bon nombre de tâches de la comptabilité générale pouvant être réalisées par le centre de gestion et l'expert-comptable. L' 'Administration générale» apparaît ensuite grâce aux trois besoins mentionnés précédemment. D'autres besoins, par contre, appartiennent aux besoins les moins ressentis (préparer la succession, établir l'organigramme et déléguer...). La «Production» puis le «Personnel» obtiennent des scores nettement inférieurs à la moyenne, ce qui était prévisible pour le personnel en raison de la faiblesse des effectifs, mais moins attendu pour la production dont le score neremonte quefaiblement pour lesseulesentreprises transformatrices. Ilest vraisemblable 
Tab. 3 Les dix besoins de formation les plus importants selon les dirigeants

\begin{tabular}{|c|c|c|c|c|c|}
\hline \multirow[b]{2}{*}{ Besoins } & \multicolumn{5}{|c|}{ Score mmoyen (max. : 5) } \\
\hline & $\begin{array}{c}\text { groupe } \\
1\end{array}$ & $\begin{array}{c}\text { groupe } \\
2\end{array}$ & $\begin{array}{c}\text { groupe } \\
3\end{array}$ & $\begin{array}{c}\text { groupe } \\
4\end{array}$ & $\begin{array}{c}\text { Ensem- } \\
\text { ble }\end{array}$ \\
\hline $\begin{array}{l}1 \text { Comment négocier efficacement avec vos } \\
\text { partenaires (fournisseurs, clients) }\end{array}$ & 3,50 & 3,50 & 4,00 & 4,25 & 3,72 \\
\hline 2 Comment payer moins d'impôts & 3,50 & 3,43 & 3,29 & 5,00 & 3,62 \\
\hline $\begin{array}{l}\text { 3a Comment déceler les attentes, les motivations et les } \\
\text { besoins de vos clients }\end{array}$ & 3,00 & 3,21 & 3,57 & 4,50 & 3,45 \\
\hline $\begin{array}{l}\text { 3b Comment déterminer les moyens publicitaires les } \\
\text { plus efficaces }\end{array}$ & $4, \infty$ & 3,43 & 3,00 & 3,75 & 3,45 \\
\hline $\begin{array}{l}\text { 5a Comment améliorer la communnication avec vos } \\
\text { clients }\end{array}$ & $4, \infty$ & 3,07 & 3,29 & 3,75 & 3,34 \\
\hline 5b Comment négocier avec votre(s) banquier(s) & 2,75 & 3,21 & 3,29 & 4,50 & 3,34 \\
\hline 7 Comment être un responsable efficace & 3,25 & 3,36 & 3,29 & 3,25 & 3,31 \\
\hline 8 Comment gérer votre trésorerie & 3,25 & 2,86 & 3,57 & 4,25 & 3,28 \\
\hline $\begin{array}{l}9 \text { Comment prévoir vos besoins d'exploitation } \\
\text { (besoins en fonds de roulement) }\end{array}$ & 3,50 & 3,43 & 3,29 & 5,00 & 3,24 \\
\hline 10a Comment prévoir la rentabilité de votre entreprise & 3,50 & 2,79 & 3,43 & 3,75 & 3,17 \\
\hline $\begin{array}{l}\text { 10b Comment fixer vos objectifs financiers et les } \\
\text { contrôles dans le temps }\end{array}$ & $3, \infty$ & 2,86 & 3,57 & 3,75 & 3,17 \\
\hline 10c Comment élaborer un plan d'action commerciale & 3,75 & 3,14 & 2,86 & 3,75 & 3,17 \\
\hline
\end{tabular}

Tab. 4. Intensité des besoins de formation par fonction

\begin{tabular}{|c|c|c|c|c|c|c|}
\hline \multirow{2}{*}{ Fonction } & \multicolumn{5}{|c|}{ Score moyen (max. : 5) } & \multirow{2}{*}{$\begin{array}{l}\text { Echant. } \\
\text { GARNIER, } \\
\text { GASSE, } \\
\text { COSSETTE }\end{array}$} \\
\hline & $\underset{1}{\text { groupe }}$ & $\begin{array}{c}\text { groupe } \\
2\end{array}$ & $\begin{array}{c}\text { groupe } \\
3\end{array}$ & $\underset{4}{\text { groupe }}$ & $\begin{array}{c}\text { Ensem- } \\
\text { ble }\end{array}$ & \\
\hline Finances & 2,73 & 2,73 & 3,65 & 4,25 & 3,16 & 2,80 \\
\hline Vente et marketing & 2,84 & 2,96 & 3,36 & 3,50 & 3,11 & 2,70 \\
\hline Comptabilité & 3,08 & 2,43 & 3,05 & 4,00 & 2,89 & 2,30 \\
\hline Administration & 2,38 & 2,52 & 2,94 & 3,19 & 2,69 & 2,85 \\
\hline Production et technique & & $\begin{array}{r}2,63 \\
2,67^{*}\end{array}$ & $\begin{array}{r}1,86 \\
2,50^{*}\end{array}$ & 2,36 & $\begin{array}{r}2,29 \\
2,57^{*}\end{array}$ & 2,30 \\
\hline Personnel & 1,44 & 2,39 & 2,71 & 2,06 & 2,26 & 2,70 \\
\hline Score global & 2,37 & 2,61 & 2,93 & 3,23 & 2,73 & 2,66 \\
\hline
\end{tabular}


transformatrices. Il est vraisemblable que les créateurs de notre échantillon, dont beaucoup ont une bonne expérience professionnelle préalable, aient résolu, au moment de la création, les problèmes de production à échelle restreinte; ce n'est que lorsqu'une certaine dimension aura été atteinte que les problèmes de production changeront de nature et se poseront de manière plus pressante.

Si l'on compare le score d'ensemble par fonction avec celui obtenu, à l'aide d'un questionnaire semblable, par B. Garnier, Y. Gasse et P. Cossette (1984) sur un échantillon de 30 firmes plus anciennes (28 ans d'existence en moyenne) et plus grandes (de 10 à 249 salariés) on remarque pour notre échantillon une intensité globale un peu plus forte $(2,73$ contre 2,66$)$, avec des besoins plus marqués en «Finances», «Marketing» et «Comptabilité» et plus faibles en «Administration» et en «Personnel». Ceci peut s'expliquer par la jeunesse de ces entreprises où les dirigeants ont une vision d'ensemble encore limitée sur l'avenir de leur affaire et ressentent l'insuffisance de leur formation dans les domaines prioritaires dans l'immédiat («Marketing» et «Finances»), tandis que la très petite taille de ces entreprises limite les problèmes de personnel.

Comme pour les problèmes de gestion, il semble que l'intensité des besoins de formation croit avec l'âge dans les fonctions «Marketing» et «Finances» et que ce parallélisme se retrouve entre problèmes de gestion et besoins de formation dans ces mêmes fonctions, ce qui justifie une analyse multi-dimensionnelle des données.

\section{Relations entre problèmes de gestion et besoins de formation}

Nous avons chiffré quatre variables de problèmes et variables de besoins pour chacune des 29 entreprises, réparties en quatre groupes d'âge et en deux secteurs, dans un tableau des données brutes. Les données brutes des 29 entreprises feront l'objet d'une analyse en deux temps.

Dans un premier temps, nous procéderons à des analyses simultanées des variables prises deux à deux : recherche d'une relation linéaire positive ou négative entre deux variables grâce au calcul de coefficients de corrélation de rang (de Spearman) et au test de STUDENT (avec un seul de signification a<0,05) pour en apprécier le degré de signification, tri croisé entre les variables significatives réalisé dans un tableau de BURT qui indique les effectifs correspondant à la même valeur de deux variables, et test du CHI-DEUX (avec un seuil de signification $a<0,1$ ) pour apprécier le degré de signification de la relation constatée.

Dans un deuxième temps à partir du croisement des modalités de toutes les variables dans le tableau de BURT, nous réaliserons une analyse factorielle des correspondances multiples. 


\subsection{Les principales relations entre variables : corrélation et tri croisé}

Les coefficients de corrélation et les CHI-DEUX retenus comme significatifs (tableau 5) confirment que l'intensité des besoins en finances augmente avec l'âge de l'entreprise, la même tendance se manifestant, mais moins nettement, pour les besoins en administration, en comptabilité et en finances; 1'âge est ainsi un facteur important d'intensification des problèmes et des besoins de formation.

Certains problèmes évoluent parallèlement : problèmes en marketing et problèmes en finances; problèmes en marketing et problèmes en production; problèmes en finances et problèmes en production. La résolution des problèmes de marketing passe ainsi par celle des problèmes financiers et celle des problèmes de production.

Quant aux besoins, pratiquement tous évoluent parallèlement, en particulier les besoins en administration, les besoins en marketing et les besoins en finances. Les besoins en production suivent les besoins en marketing, de bonnes ventes ne pouvant se faire qu'avec des produits et un processus de production performants. Les besoins en production et les besoins en personnel apparaissent liés, la production à une certaine échelle demandant un personnel formé et compétent.

Enfin, il semble que l'apparition de problèmes dans certains domaines entraîne une demande de formation dans ce même domaine : c'est le cas pour le marketing et la finance. En outre, des problèmes en marketing paraissent aussi à l'origine d'une demande de formation en finances et comptabilité; il ne faut pas oublier que les problèmes sont liés en marketing et finances, mais c'est le marketing qui est prédominant. Des difficultés commerciales mettent en péril un équilibre financier, déjà fragile à l'origine d'une entreprise et permettent de prendre conscience de l'importance de la finance et de la nécessité d'une bonne formation financière et comptable.

\subsection{Analyse factorielle des correspondances multiples entre les variables}

L'analyse fait apparaître plusieurs axes factoriels. Leur étude permet de classer les entreprises en fonction de leurs problèmes et de leurs besoins.

Les trois premiers axes représentent respectivement $13,71 \%, 13,11 \%$ et $11,40 \%$ de l'inertie du nuage, soit un cumul de $38,22 \%$; les autres axes voient leur intertie chuter rapidement $(7,82 \%, 7,20 \%, 6,12 \%$...) et ne fournissent plus de renseignements intéressants; aussi, limiterons-nous nos commentaires aux trois premiers axes. Ces axes sont essentiellement des axes de besoins. Avant de les analyser, regardons l'évolution des modalités des variables les plus significatives dans le plan factoriel $1 \times 2$ (graphique 1 p. 341). 


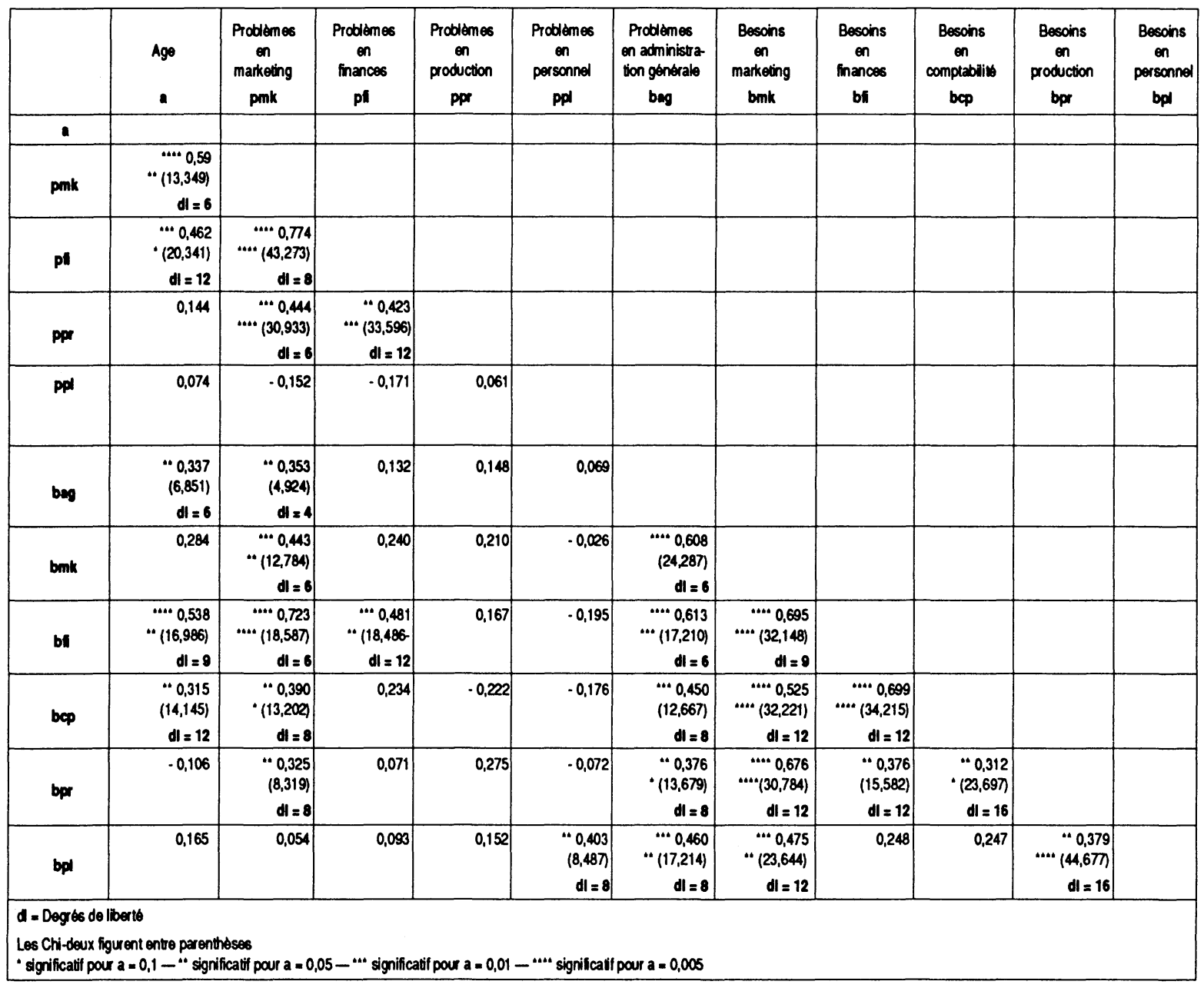


Les variables de besoins les plus contributives de l'axe 1 (bfi, bmk, bag) décrivent des trajectoires en forme de croissant orientées vers le plan inférieur gauche où l'on trouve l'âge 4 (a4); les variables de problèmes les plus contributives (pmk, pfi) décrivent elles aussi un croissant mais orienté vers le plan supérieur gauche où se trouve l'âge 3 (a3): il apparaît ainsi un décalage entre l'intensité des problèmes qui est maximum pour l'âge 3 et l'intensité des besoins qui est maximum pour l'âge 4;

\subsubsection{Le premier axe factoriel (graphique 1)}

Les modalités les plus contributives sont réparties d'une part sur la partie positive de l'axe (bmk2, bfi2, bag2, pmk2, pfi2, a1) et, d'autre part, sur la partie négative de l'axe (bfi5, bmk5, bcp5, bag4, bpr5, bp15, pmk3, pfi4, a4). L'axe 1 sépare ainsi nettement les entreprises très jeunes (a1), à faibles problèmes en marketing et en finances (pmk2, pfi2) et aux besoins de formation limités aussi bien en administration qu'en marketing et finances (bag2, bmk2, bfi2), et les entreprises les plus anciennes (14), aux problèmes plus marqués en marketing et finances (pmk3, pfi4), et aux besoins de formation les plus marqués, aussi bien dans les domaines de l'administration, du marketing et des finances (bag4, bmk5, bfi5, bcp5), que dans ceux de la production et du personnel (bp5, bpl5).

\subsubsection{Le deuxième axe factoriel (graphique 1)}

Le deuxième axe voit lui aussi les modalités les plus significatives figurer sur la partie positive et sur la partie négative; il distingue ainsi les entreprises d'âge 3 (a3) des entreprises d"'âge 4 (a4). Les entreprises d"âge 3 ont souvent des problèmes plus marqués en marketing, en finances et en production (pmk4, pfi5, ppr4) que celles d"âge 4, mais des besoins de formation moins pressants (bmk4, bp13,bpr3,pfi4). Comme le laissaient pressentir les trajectoirescomparées d"évolution des problèmes et des besoins, il apparaît que la demande de formation suit avec un certain décalage l"apparition des problèmes. Les plus typiques entreprises d"âge 3 sont des entreprises de production (sct2), ce qui explique la plus forte intensité des problèmes de production (ppr4).

\subsubsection{Le troisième axe factoriel (graphique 2)}

Les modalités les plus significatives se trouvent sur la partie négative de l'axe soit à droite, soit à gauche du graphique. Le troisième axe isole les entreprises d'âge 2; en général (à droite sur le graphique), ces entreprises rencontrent peu de problèmes en marketing, finances, production et personnel (pmk2, pfi2, ppr2, ppl2) et ressentent des besoins de formation limités dans ces domaines (bmk2, pfi2, ppr2, pp12) ainsi qu'en administration et en comptabilité (bag2, bcp2). Mais il y a des exceptions (partie gauche du graphique) qui, malgré des problèmes limités, ont une 


\section{Graphique 1 Plan factoriel $1 \times 2$}

\section{Les variables}

PLAN DE PROJECIION DES SI POINTS SUR LES AXES 1 ET 2

AXE 1 MHORIZONTAL GXE 2 NEERTICAL

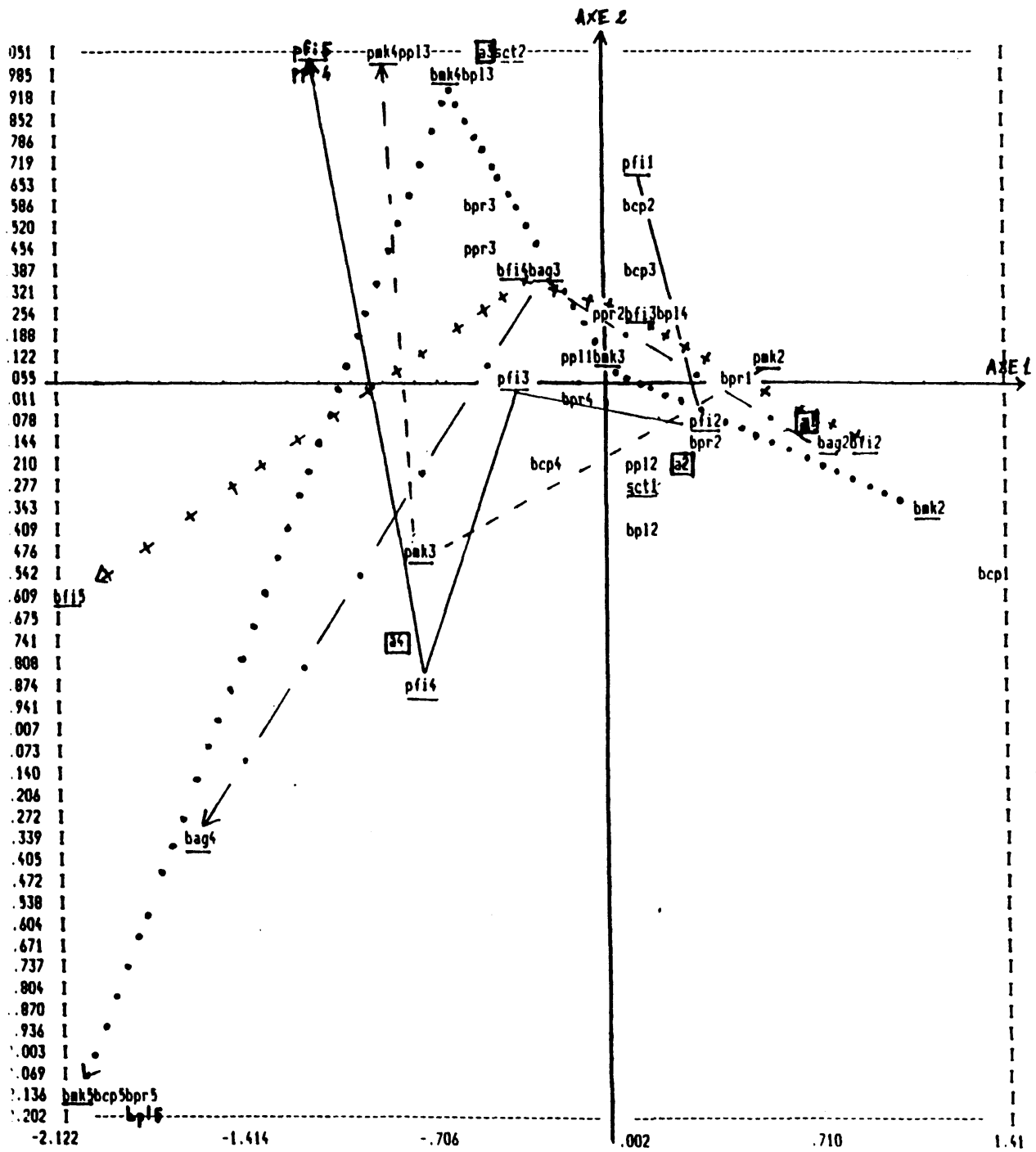


Graphique 2 Plan factoriel $1 \times 3$

\section{Les variables}

PLAN DE PROJECTION DES SI POINTS SUR LES AXES 1 EI 3

AXE I /HORIZONTAL AXE 3 /VERTICAL

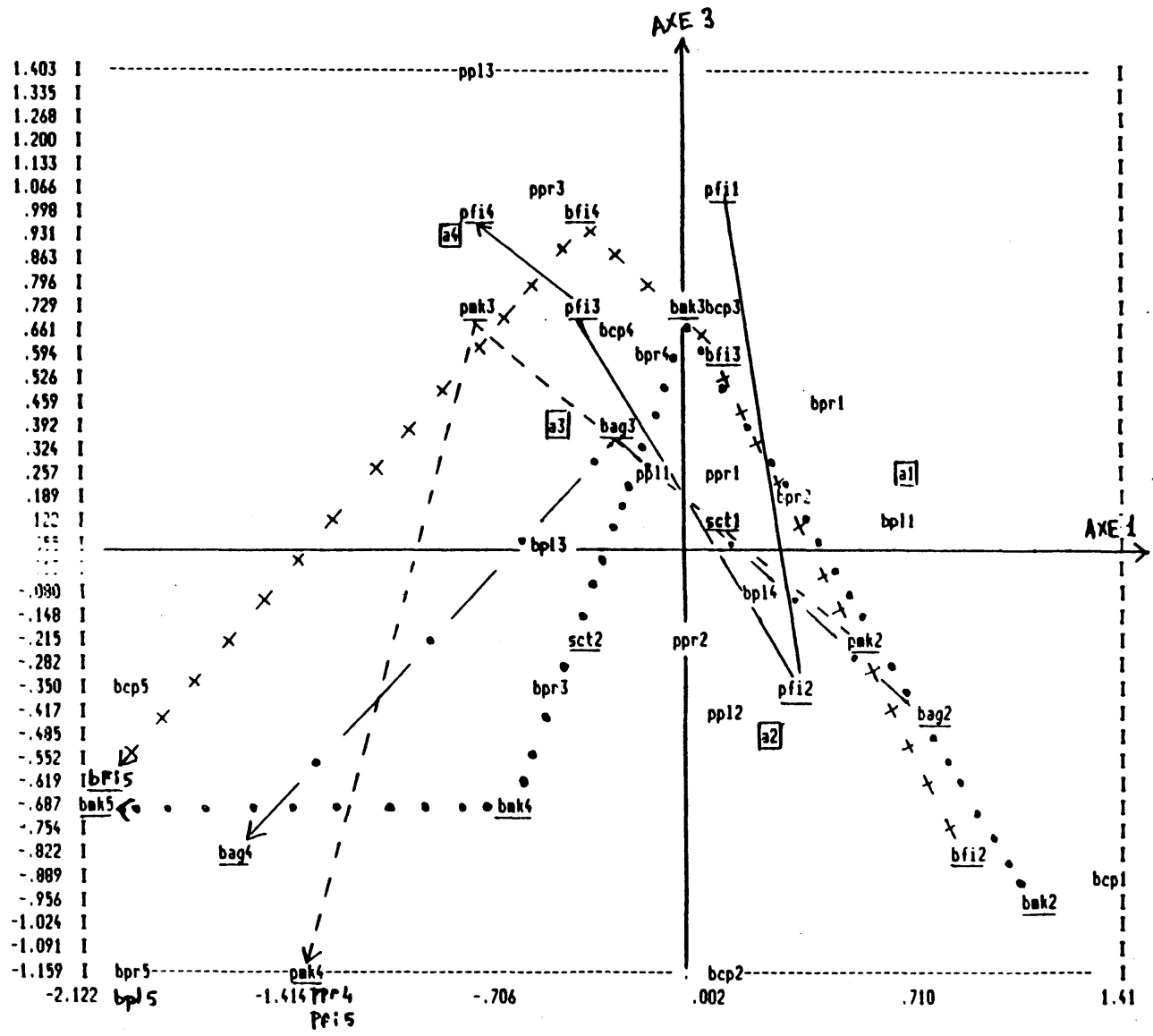


forte demande de formation (bfi5, bmk5, bpr5, bpl5) qui les rapproche des entreprise d'âge 4 . selon leur âge.

L'analyse factorielle fait ainsi apparaître les caractéristiques des entreprises

Les entreprises de moins de six mois (âge 1) ont résolu leurs problèmes immédiats de gestion pour pouvoir démarrer leur activité et expriment peu de besoins de formation, tout leur temps étant occupé par la gestion courante de leurs affaires.

Les entreprises de six mois à un an (âge 2) rencontrent encore peu de problèmes et lorsque les problèmes s'intensifient un peu, c'est surtout dans les domaines du marketing et de la finance, mais les difficultés en production et en personnel peuvent apparaître, en particulier à l'occasion des premières embauches. Les demandes de formation sont un peu plus marquées, mais restent limitées; cependant, certaines entreprises expriment déjà une forte demande de formation liée à l'augmentation des difficultés.

C'est entre la première et la deuxième année (âge 3 ) que les problèmes s'accentuent en marketing et en finances, mais aussi en production et en personnel, pour les entreprises industrielles transformatrices, soit lorsqu'elles abordent la production à plus grande échelle. Les entrepreneurs prennent alors pleinement conscience que les besoins de formation s'accroissent corrélativement, en particulier les besoins en production et en personnel.

Les entreprises de plus de deux ans (âge 4) n'indiquent pas de problèmes plus intenses que ceux des entreprises de un à deux ans, mais ont une demande plus pressante de formation : il apparaît ainsi un décalage, pouvant atteindre un an, entre l'apparition des difficultés et la demande de formation. Ce retard correspond au temps nécessaire à la prise de conscience de la nécessité d'une formation approfondie pour gérer efficacement une organisation dont la complexité s'intensifie.

\section{Conclusion}

H. Mahe (1984) remarquait des réticences dans la demande de formation de la part des dirigeants-propriétaires des petites entreprises. Ces réticences semblent provenir principalement du manque de disponibilité temporelle des dirigeants, en raison de leur charge de travail, ainsi que d'offres de formation souvent lourdes et standardisées.

Seules des enquêtes directes auprès des dirigeants peuvent faire apparaitre la nature et l'intensité des besoins de formation, en dehors des demandes d'urgence lorsque la situation de l'entreprise se détériore. Pour les organismes de formation, c'est la connaissance des besoins précis aux différents stades de l'évolution des entreprises qui permettra de mettre au point des modalités de formation adéquates. 
Les dirigeants que nous avons interrogés sont informés sur les principaux problèmes de gestion rencontrés par la TPE en démarrage et sur les possibilités de formation complémentaire. Il s'agit pour l'organisme de formation (le C.P.F.C.E. ici) de proposer au moment opportun des actions de formation appropriées. La demande des dirigeants interrogés porte essentiellement sur des formations courtes (ne dépassant pas 15 heures), réparties en demi-journées le matin en début de semaine, sous forme de discussions à partir de problèmes similaires rencontrés par les participants, c'est-à-dire sur une formation par groupes de travail.

\section{BIBLIOGRAPHIE}

Capiez, A., (1988), «Conditions d'émergence des T.P.E. et P.E.: l'exemple du Maine et Loire (France)», Revue Internationale P.M.E., vol. $1, \mathrm{n}^{\circ} 2$.

Capron, H., Durez, M. et Lux, B., (1987), «Les problèmes de gestion des PME en Belgique : les résultats d'une enquête», Revue P.M.O., vol. $3, \mathrm{n}^{\circ} 1$.

Churchill, N.C. et Lewis, V.L., (1983), «Les cinq stades d'évolution d'une PME», Harvard, l'Expansion, automne.

D'Amboise, G. et Parent, A., (1988), «Les problèmes des petites entreprises : ce que révèlent les études nord-américaines», communication au Colloque International de Montpellier, mai.

Deeks, J., (1976), The Small Owner Manager : Entrepreneurial Behavior and Management Practice, New York, Preager Publishers.

Fourcade, C., (1988), «Structuration du système productif régional par système de petites unités», communication au Colloque International de Montpellier, mai.

Garnier, B., Gasse, Y. et Cossette, P., (1984), «Les besoins de formation des dirigeants de P.M.E.: une étude empirique au Québec», Enseignement et gestion, $\mathrm{n}^{\circ} 31$, automne.

Hirigoyen, G., (1984), Contribution à la connaissance des comportementsfinanciers des PME familiales, thèse de doctorat, Bordeaux.

Lorrain, J. et Dussault, L., (1988), «Les difficultés, les solutions et la gestion : cas d'entreprises manufacturières en phase de survie et d'établissements, communication au Colloque International de Montpellier, mai.

Mahe, H., (1984), «P.M.E. en démarrage : l'apprentissage de la direction», Enseignement et gestion, $\mathrm{n}^{\circ} 31$, automne.

Mahe, H., (1985), «P.M.E. en démarrage, du décideur au gestionnaire», Enseignement et gestion, $\mathrm{n}^{\circ} 36$, hiver.

Marchesnay, M., (1986), La stratégie, du diagnostic à la décision industrielle, Paris, Chotard et associés.

Marchesnay, M., (1988), «Sur les relations petite entreprise-environnement local : quelques observations positives et normatives», communication au Colloque International de Montpellier, mai.

Strazzieri, A., «Études sur les besoins des chefs d'entreprise en matière de formation : résultats d'une enquête», I.A.E., Aix-en-Provence.

Thomas, J. et Sireno, P., (1980), «Training needs of managers of small manufacturing firms», American Journal of Small Business, vol. 4, $\mathrm{n}^{\circ} 3$, hiver.

Ouvrage collectif, (1988), Économie et gestion de la petite entreprise, Paris, Vuibert. 